\title{
The Complexation of Protonated Peptides with Saccharides in the Gas Phase Decreases the Rates of Hydrogen/Deuterium Exchange Reactions
}

\author{
M. Kirk Green, Sharron G. Penn, and Carlito B. Lebrilla \\ Department of Chemistry, University of California, Davis, California, USA
}

\begin{abstract}
Gas-phase noncovalently bound complexes are probed by hydrogen/deuterium exchange. The complexes, composed of a protonated amino acid and a monosaccharide, are investigated to observe the effects of complexation on the rates of exchange. Rate constants are determined and compared for complexed and uncomplexed amino acids. The overall rate constant, which corresponds to exchange of a specific number of hydrogens, is deconvoluted to yield site-specific rate constants. Complexation of amino acids with saccharides significantly decreases the rate constants of the exchange. Results of molecular orbital calculations are provided to explain the decrease in the rates. (J Am Soc Mass Spectrom 1995, 6, 1247-1251)
\end{abstract}

$\mathrm{T}$ The investigation of gas-phase noncovalently bound complexes has been made possible by recent developments of ionization sources for macromolecules. The isolation of these complexes in the gas phase provides supporting evidence for their formation in the solution phase [1-6]. Hydrogen/ deuterium $(\mathrm{H} / \mathrm{D})$ exchange offers a nondestructive and low energy probe of the complexes' structure [7-9]. It has been used already to characterize folding in complex gas-phase proteins [10,11]. Development of this probe for noncovalently bound complexes, however, requires a deeper understanding of the mechanism and factors that affect the rates. In amino acids and peptides, structural and energetic factors affect the rates of $\mathrm{H} / \mathrm{D}$ exchange [12]. For example, the rates of exchange for carboxylic acid hydrogens differ greatly from amine hydrogens $[9,13]$. In this communication, we report that in complexes that involve a protonated peptide and a sugar moiety, the rates of $H / D$ exchange decrease, relative to the lone protonated species, even when the number of labile hydrogens significantly increases. For this study, a new method was developed to calculate the rate constants by deconvolution of the values for each exchangeable hydrogen. Evidence for a unique, intracomplex hydrogen exchange between the peptide and the sugar is provided also. Additionally, to our knowledge this is the first report of gas-phase $H / D$ exchange that involves proton bound complexes.

The mechanism of gas-phase $H / D$ exchange depends on the difference in gas-phase basicity of the

Address reprint requests to Dr. Carlito $B$. Lebrilla, Department of Chemistry, University of California--Davis, Davis, CA 95616. deuterated reagent and the conjugate base of the protonated species $[9,13]$. For compounds that contain a single functional group, gas-phase basicities of less than $80-\mathrm{kJ} / \mathrm{mol}$ difference between the protonated species and the deuterated reagent promote exchange [8]. When the gas-phase basicities (GBs) are similar, $\mathrm{H} / \mathrm{D}$ exchange occurs by simple proton transfer possibly through a four center intermediate. With significantly larger differences in GB, the contribution by this direct mode is decreased. For this reason, $H / D$ exchange does not occur between deuterated methanol (GB $728.8 \mathrm{~kJ} / \mathrm{mol}$ ) and protonated amines (GB $\sim 800-900 \mathrm{~kJ} / \mathrm{mol})[9,14]$. When the protonated substrate contains two or more functional groups, exchange again is observed even with GB differences of greater than $160 \mathrm{~kJ} / \mathrm{mol}[9,12,13,15]$. We have attributed this reactivity to the simultaneous interaction between two functional groups and the incoming deuterated reagent (as shown with protonated glycine and $\mathrm{CH}_{3} \mathrm{OD}$ ) that produces a "cooperative interaction" suitable for $H / D$ exchange [13].

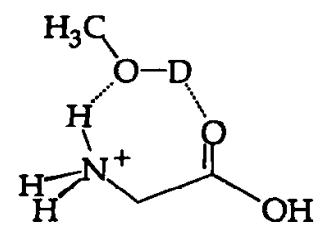

Molecules that can produce this interaction with the deuterating reagent also can have strong intramolecular interactions. The rate constant of intermolecular 
$\mathrm{H} / \mathrm{D}$ exchange is known to increase almost linearly as the difference in the proton affinity of the two most basic sites in amino acids and peptides decreases [13]. As a consequence, $H / D$ exchange can be used to probe intramolecular interactions in protonated species. Intermolecular interactions in complexes (intracomplex interactions) are good candidates to probe with $\mathrm{H} / \mathrm{D}$ exchange.

\section{Experimental}

In the experiments performed for this report, mixed dimer ions that consist of amino acids and monosaccharides were produced by liquid secondary ion mass spectrometry ionization by using an external source Fourier transform instrument [16]. The H/D exchange experiments were performed by trapping the ionic species in a background pressure of $\mathrm{CH}_{3} \mathrm{OD}(2-4 \times$ $10^{-7}$ torr) and monitoring the intensities of the peaks that correspond to different numbers of deuteriums incorporated as functions of time. An earlier method of data treatment used in this laboratory treated the system as series of successive reactions,

$$
\mathrm{CH}_{3} \mathrm{OD}+\left[\mathrm{H}_{n}\right]^{+} \underset{k_{-1}}{\stackrel{k_{1}}{\rightleftharpoons}} \mathrm{CH}_{3} \mathrm{OH}+\left[\mathrm{H}_{n-1} \mathrm{D}\right]^{+}
$$

where $n$ represents the number of deuteriums incorporated, and calculated the apparent rate constants $k_{n}$ by solution of the associated set of coupled differential equations and iteration to the best fit to the experimental ion intensities [9, 13]. Thus the values $k_{1}, k_{2}$, $k_{3}, \ldots, k_{n}$, where $n$ is the total number of hydrogens exchanged, represent the rate constants for the respective exchange of one, two, and so forth hydrogens. In reality, these rate constants are combinations of rate constants made up of $n$ exchange sites. This treatment now has been modified to deconvolute the rate constants for the individual sites. In the new treatment, the system has been treated as a series of independent reactions,

$$
S_{i}(\mathrm{H})+\mathrm{CH}_{3} \mathrm{OD} \underset{k_{-i}}{\stackrel{k_{1}}{\rightleftharpoons}} S_{i}(\mathrm{D})+\mathrm{CH}_{3} \mathrm{OH}
$$

where $S_{j}$ represents the $j$ th exchange site. The specific rate constants, $k_{\mathrm{i}}, k_{\mathrm{ij}}, k_{\mathrm{iii}}, \ldots, k_{n}$, are determined by solution of the corresponding set of independent dif- ferential equations to obtain fractional occupancies of each site. Use of the appropriate combinations and permutation of the fractional occupancies yields expected ion intensities, which are then fitted to the experimental data. With this method and some structural insight, we can assign rate constants to specific sites.

\section{Results and Discussion}

The rate constants for the exchange of protonated glycine, glycerol, and the mixed dimer are tabulated (Table 1). The values are normalized to the largest rate constant of protonated glycine. The small face numbers correspond to the uncertainties in the values. Uncertainties in the rate constants are determined by the standard deviation in the fit. For each species the rate constants are listed in decreasing order. Protonated glycine has four exchangeable hydrogens, one on the carboxylic acid hydrogen and three on the terminal amine. In earlier studies, we noted that the first exchange in protonated amino acids without basic side chains occurs primarily on the carboxylic oxygen [13]. The new constants reflect these earlier observations. Site $i$, therefore, corresponds to the carboxylic acid and the rate constant is 10 times greater than exchanges on sites ii-iv, which correspond to the three equivalent amino hydrogens. Protonated glycerol exchanges rapidly with four rate constants that are identical, which indicates that the three hydroxyl hydrogens and the proton are all chemically equivalent.

An increase in the number of labile hydrogens does not necessarily increase the reactivity of the gas-phase species. The complex composed of protonated glycine and glycerol has rate constants that are considerably lower than either protonated monomer. The largest value, 13.4, is similar in magnitude to the three slower rates of protonated glycine. There could be significant errors $(50 \%)$ in the absolute values of the given rate constants due to a background of undeuterated methanol and errors in the calibration of the ion gauge tube. This error should be the same in each determination so that the relative errors (given in parentheses in the tables) are significantly smaller $[9,13]$. The remainder of the rate constants are significantly smaller. In the mass spectrum, the perdeuterated product is observed, but only after $40 \mathrm{~s}$ with a background pressure of $3.1 \times 10^{-7}$ torr, compared to about $5 \mathrm{~s}$ for glycine under similar conditions. The similarity of the rate

Table 1. Summary of $\mathrm{H} / \mathrm{D}$ exchange reactions between $\mathrm{CH}_{3} \mathrm{OD}$ and protonated glycine, protonated glycerol, and the

\begin{tabular}{|c|c|c|c|c|c|c|c|}
\hline Species & $k_{\mathrm{i}}$ & $k_{i i}$ & $k_{\mathrm{iii}}$ & $k_{\mathrm{iv}}$ & $k_{v}$ & $k_{\mathrm{vi}}$ & $k_{\text {vii }}$ \\
\hline$\overline{\mathrm{GH}^{+}}$ & $100(6)$ & $10.7(3.2)$ & $10.7(3.2)$ & $10.7(3.2)$ & & & \\
\hline $\mathrm{YH}^{+}$ & $225(38)$ & $225(38)$ & $225(38)$ & $225(38)$ & & & \\
\hline $\mathrm{GH}^{+} \ldots \mathrm{Y}$ & $13.4(1.9)$ & $3.0(0.8)$ & $3.0(0.8)$ & $3.0(0.08)$ & $3.0(0.8)$ & $3.0(0.8)$ & $3.0(0.8)$ \\
\hline
\end{tabular}
protonated mixed dimer ${ }^{\circ}$

\footnotetext{
'Rate constants are normalized to $k$ of protonated glycine and are listed in italics. Numbers in parentheses are relative deviation of the values. Absolute rate constant $k_{i}($ glycine $)=62.8 \times 10^{-12}$ molecules $/ \mathrm{cm}^{3} \mathrm{~s} . \mathrm{G}=$ glycine; $Y=g l y c e r o l$.
} 
constants $k_{\mathrm{ii}}-k_{\mathrm{vii}}$ is probably not significant because the values are small with large uncertainties.

The role of stereochemistry in the H/D exchange of the complex was investigated by complexation of glycine to four different monosaccharides (see Chart 1). The three pyranose (glucose, galactose, and mannose) and one furanose (fructose) sugars examined differ by ring size and relative orientation of the hydroxyl groups. Because coordination between the sugar and the amino acid is expected to occur through the hydroxyl groups, the relative stereochemistry may affect specific rate constants. Table 2 lists the rate constants for the eight hydrogens observed to exchange in the protonated dimer. Complexation appears to severely hinder all rates of exchange when either glycine or alanine is complexed to sugars, in the same manner observed for glycine complexed with glycerol. A moderately fast exchange occurs at a single site with rate constants that range between 11.3 and 17.0. The differences in these values probably are not significant. The exchanges that correspond to $k_{\mathrm{ii}}-k_{\mathrm{viii}}$ are very slow, although most labile hydrogens do exchange to some extent. There are variations in the rate constants that depend on the sugar. However, the numbers are too small to conclude that the variations are due to stereochemistry. It is possible that with larger sugars and peptides, one may observe variations in rate constants due to stereochemistry.

The large rate constants that accompany strong intramolecular interactions also are observed with protonated lysine and diglycine (Table 3 ). Protonated lysine undergoes $H / D$ exchange with six equivalent rate constants that correspond to the protonated amine hydrogens (five) and the carboxylic acid. There appears to be no distinction between the two types of hydrogens. The large number of moderately large rate constants allows rapid exchange of all hydrogens with deuterium in the protonated molecule. Similarly, protonated diglycine exchanges rapidly with three rate constants that are equal and large (158), one that is moderate (24), and one that is small (7.8). On the basis of our understanding of $\mathrm{H} / \mathrm{D}$ exchange, we propose that these values are for the three amine hydrogens (protonated), the acid hydrogen, and the amide hydrogen, respectively.

Complexation of both protonated lysine and diglycine greatly diminishes the rates of exchange. The
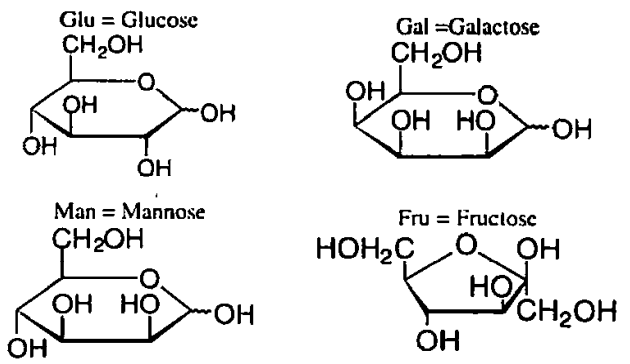

Chart 1.

complex of protonated lysine with glucose exhibits only two slow exchanges (relative rate constants of 5.1 and 6.1) during the same time as protonated lysine exchanges to form the perdeuterated product. The complex of protonated diglycine with glucose has three very slow exchange rates with equivalent rate constants of 0.3. Interestingly, although protonated lysine and diglycine monomers have overall greater rate constants than either protonated glycine or alanine, the corresponding rate constants for the complexes with the sugars are considerably smaller.

The one fast rate in the complexes of glycine and alanine mirrors a similar fast rate in the uncomplexed protonated amino acid. This points to the possibility that the fastest rate still may correspond to the exchange of the acid hydrogen. If this is the case, then the reactions of the esters should not have the single fast rate leaving only the slow rates, as has been reported for the reactions of protonated glycine and alanine esters $[9,13]$. The reactions of glycine ester complexed to glycerol and glucose and alanine ester complexed to glucose were performed under similar conditions as those for the other complexes. No deuterium incorporation was obtained during the same reaction times.

$$
\begin{aligned}
\mathrm{GE} \cdots \mathrm{H}^{+} \cdots \mathrm{Y}+\mathrm{CH}_{3} \mathrm{OD} \rightarrow \text { no reaction } \\
\mathrm{GE} \cdots \mathrm{H}^{+} \cdots \mathrm{Clu}+\mathrm{CH}_{3} \mathrm{OD} \rightarrow \text { no reaction } \\
\mathrm{AE} \cdots \mathrm{H}^{+} \cdots \mathrm{Glu}+\mathrm{CH}_{3} \mathrm{OD} \rightarrow \text { no reaction } \\
\mathrm{GE}=\text { glycine methyl ester } \\
\mathrm{AE}=\text { alanine methyl ester } \\
\mathrm{Y}=\text { glycerol }
\end{aligned}
$$

Table 2. Summary of $\mathrm{H} / \mathrm{D}$ exchange reactions between $\mathrm{CH}_{3} \mathrm{OD}$ and protonated glycine complexed to monosaccharides"

\begin{tabular}{lcccccccc}
\hline Species & $k_{\mathrm{i}}$ & $k_{\mathrm{ii}}$ & $k_{\mathrm{iii}}$ & $k_{\mathrm{iv}}$ & $k_{\mathrm{v}}$ & $k_{\mathrm{vi}}$ & $k_{\mathrm{vii}}$ & $k_{\mathrm{vii}}$ \\
\hline \hline $\mathrm{GH}+\cdots \mathrm{Glu}$ & $17.0(2.4)$ & $2.4(2.4)$ & $2.4(2.7)$ & $0.8(2.5)$ & $0.8(1.4)$ & $0.8(1.1)$ & $0.8(1.1)$ & $0.8(0.8)$ \\
$\mathrm{GH}^{+} \cdots \mathrm{Gal}$ & $12.9(0.6)$ & $1.3(0.6)$ & $1.3(0.6)$ & $1.3(0.6)$ & $1.3(0.6)$ & $1.3(0.6)$ & $0.5(0.3)$ & $0.5(0.3)$ \\
$\mathrm{GH}^{+} \cdots$ Man & $11.3(0.5)$ & $2.2(0.2)$ & $0.2(0.2)$ & $0.2(0.2)$ & $0.2(0.2)$ & $0.2(0.2)$ & $0.2(0.2)$ & $0.2(0.2)$ \\
$\mathrm{GH}^{+} \ldots$ Fru & $13.1(0.8)$ & $1.1(0.3)$ & $0.3(0.2)$ & $0.3(0.2)$ & $0.3(0.2)$ & $0.3(0.2)$ & $0.3(0.2)$ & $0.3(0.2)$ \\
$\mathrm{AH}^{+} \cdots \mathrm{Glu}$ & $14.6(0.5)$ & $4.3(0.3)$ & $0.5(0.2)$ & $0.5(0.2)$ & $0.5(0.2)$ & $0.5(0.2)$ & $0.5(0.2)$ & $0.5(0.2)$ \\
\hline
\end{tabular}

\footnotetext{
a Rate constants are normalized to $k_{i}$ of protonated glycine and are listed in italics. Numbers in parentheses are relative deviation of the values.
} 
Table 3. Summary of $\mathrm{H} / \mathrm{D}$ exchange reactions between $\mathrm{CH}_{3} \mathrm{OD}$ and protonated Diglycine and Lysine complexed with glucose"

\begin{tabular}{lcccccc}
\hline Species & $k_{\mathrm{i}}$ & $k_{\mathrm{ii}}$ & $k_{\mathrm{ii1}}$ & $k_{\mathrm{iv}}$ & $k_{\mathrm{v}}$ & $k_{\mathrm{vi}}$ \\
\hline \hline $\mathrm{LysH}^{+}$ & $20.0(5.1)$ & $20.0(5.1)$ & $20.0(5.1)$ & $20.0(5.1)$ & $20.0(5.1)$ & $20.0(5.1)$ \\
$\mathrm{LysH}^{+} \ldots \mathrm{Glu}$ & $5.7(3.7)$ & $6.1(3.7)$ & $*$ & $*$ & $*$ & $*$ \\
$\mathrm{G}_{2} \mathrm{H}^{+}$ & $158(25)$ & $158(25)$ & $158(25)$ & $24(2)$ & $7.8(0.8)$ & $*$ \\
$\mathrm{G}_{2} \mathrm{H}^{+} \ldots \mathrm{Glu}$ & $0.3(0.2)$ & $0.3(0.2)$ & $0.3(0.2)$ & $*$ & $*$ & $*$ \\
\hline
\end{tabular}

${ }^{a}$ Rate constants are normalized to $k_{i}$ of protonated glycine and are listed in italics. Numbers in parentheses are relative deviation of the values. The asterisk $(*)$ indicates an exchangeable hydrogen but no exchange observed.

The total lack of reactivity in the ester complexes is a strong indication that the acid hydrogen plays a central role in the $H / D$ exchange reactions. It suggests further that exchange in the corresponding acid complex occurs only through the acid hydrogen. If all exchanges between $\mathrm{CH}_{3} \mathrm{OD}$ and the complex occur through the acid hydrogen, then the incorporation of more deuterium, which results in exchange of the amine and hydroxyl hydrogens, is likely the result of intracomplex H/D exchange. The attachment of monosaccharides to the amino acids involves a complex interaction, but the glycine-glycerol complex probably involves interaction of the protonated amine with the hydroxyl groups of glycerol.

We have performed molecular orbital (MO) calculations by using AM1 on the intermediate described (1; Scheme I) and have found it to be stable on the AM1 $[17,18]$ surface. The heat of dimerization (that involves a protonated glycine and a neutral glycerol) is predicted to be $-101.3 \mathrm{~kJ} / \mathrm{mol}$. The interaction effectively solvates the protonated terminal amine, as evidenced by the short $\mathrm{O} \cdots \mathrm{H}(\mathrm{N})$ bond distances $(\sim 2.1 \AA)$. H/D exchange occurs when the trimer with $\mathrm{CH}_{3} \mathrm{OD}$ is formed (2, Scheme I). This reaction is exothermic by $-49.0 \mathrm{~kJ} / \mathrm{mol}$, but is energetically incapable of dissociating the original protonated dimer. Exchange proceeds through the intermediate 2 when the amine and the carboxylic acid cooperate to bind the incoming $\mathrm{CH}_{3} \mathrm{OD}$, as in the case of protonated glycine. On the AM1 surface, a hydroxyl group of glycerol moves away from the protonated amine when the hydroxyl group of methanol is attached.

The specific coordination of the dimer complex may change before or during the formation of the nascent trimer. The rate of $H / D$ exchange can be decreased by the hydroxyl group of glycerol that blocks the site where $\mathrm{CH}_{3} \mathrm{OD}$ can exchange (4). The differences in reaction enthalpy, due to complexation of $\mathrm{CH}_{3} \mathrm{OD}$, between the complex and the lone protonated species

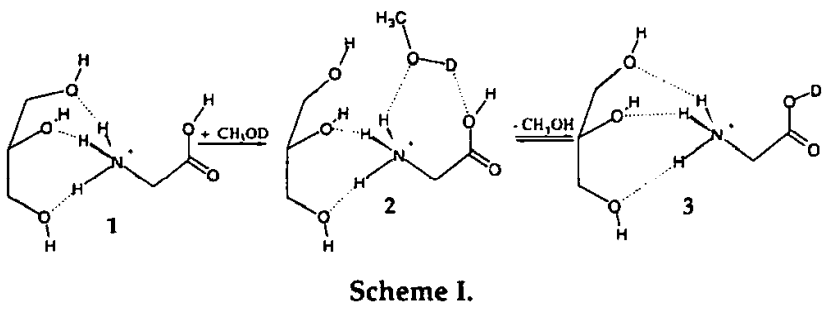

do not account for the differences in reactivity. Attachment of methanol to protonated glycine is only 13.4 $\mathrm{kJ} / \mathrm{mol}$ more exothermic than to the protonated dimer. The intermediate 4 , or one similar, also can account for the intracomplex $\mathrm{H} / \mathrm{D}$ exchange because the binding of the hydroxyl group can be similar to that of methanol.

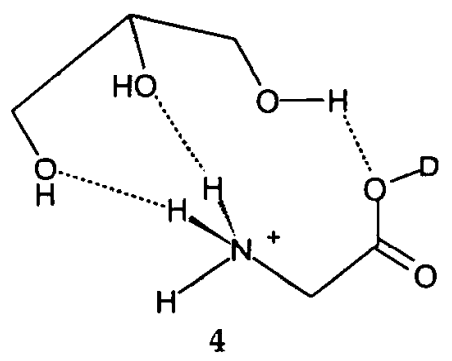

The affect of complexation on the H/D exchange of macromolecules will depend on where the protons (in multiply charged species) are incorporated in the complex. If the protons are incorporated deep within the complex and are sterically blocked, then H/D exchange can be extremely slow or nonexistent. This behavior also is observed in the H/D exchange of protein-ligand complexes in solution [19]. If the protons are on the surface of the complex, then $\mathrm{H} / \mathrm{D}$ exchange can be rapid. In these situations, H/D exchange will be a useful probe of the complex surface.

\section{Acknowledgments}

Funding was provided by the National Science Foundation (CHE-9310092). The computers for performing $\mathrm{MO}$ calculations were provided by the Department of Chemistry, U.C. Davis.

\section{References}

1. Smith, R. D.; Light-Wahl, K. J. Biol. Mass Spectrom. 1993, 22, 493-501.

2. Katta, V.; Chait, B. T. J. Am. Chem. Soc. 1991, 113, 8534-8535.

3. Ganem, B.; Li, Y.-T.; Henion, J. D. J. Am. Chem. Soc. 1991, 113, 6294-6296.

4. Ganguly, A. K.; Pramanik, B. N.; Tsarbopoulos, A.; Covey, T. R.; Huang, E.; Fuhrman, S. A. J. Am. Che'm. Soc. 1992, 114, $6559-6560$.

5. Ogorzalek-Loo, R. R.; Goodlett, D. R.; Smith, R. D.; Loo, J. A 1. Am. Chem. Soc. 1993, 115, 4391-4392. 
6. Light-Wahl, K. J.; Springer, D. L.; Winger, B. E.; Edmonds, C. G.; Camp, D. G.; Thrall, B. D.; Smith, R. D. J. Am. Chem. Soc: 1993, 115, 803-804.

7. Ranasinghe, A.; Cooks, R. G.; Sethi, S. K. Org. Mass Spuctrom. 1992, 27, 77-88.

8. Lias, S. I. Physs. Chom. 1984, 88, 4401-4407.

9. Gard, E.; Green, M. K.; Bregar, J.; Lebrilla, C. B. Org. Mass Spectrom. 1993, 28, 1632-1639.

10. Winger, B. E.; Light-Wahl, K. J.; Rockwood, A. L.; Smith, R. D. I. Am. Chem. Soc. 1992, 114, 5897-5898.

11. Suckau, D.; Shi, Y.; Beu, S. C.; Senko, M. W.; Quinn, J. P.; Wampler, F. M., III; Mclafferty, F. W. Proc. Natl. Acad. Sci. USA 1993, 90, 790-793.

12. Campbell, S.; Rodgers, M. T.; Marzluff, E. M.; Beauchamp, J. L. I. Am. Chr'm. Soc. 1994, 116, 9765-9766.
13. Gard, E.; Green, M. K.; Bregar, J.; Lebrilla, C. B. J. Am. Soc. Mass Spectrom. 1994, 5, 623-631.

14. Lias, S. G.; Liebman, J. F.; Levin, R. D. J. Pliys. Chem. Ref. Data 1984, 13, 695-808.

15. Cheng, X.; Fenselau, C. Int. I. Mass Spectrom. Ion Proccesses 1992, 122, 109-119.

16. McCullough, S. M.; Gard, E.; Lebrilla, C. B. Int. I. Mass Spectrom. Ion Processes 1991, 107, 91-102.

17. Dewar, M. J. S.; Zoebisch, E. G.; Healy, E. F.; Stewart, J. J. P. J. Am. Chem. Soc. 1985, 107, 3902-3909.

18. Dewar, M. J. S.; Dieter, K. M. J. Am. Chem. Soc. 1986, 108, 8075-8086.

19. Anderegg, R. J.; Wagner, D. S. 1. Am. Clem. Soc. 1995, 117, 1374-1377. 